\title{
Legendre Wavelets Method for Solving Fractional Population Growth Model in a Closed System
}

\author{
M. H. Heydari, ${ }^{1}$ M. R. Hooshmandasl, ${ }^{1}$ C. Cattani, ${ }^{2}$ and Ming Li $^{3}$ \\ ${ }^{1}$ Faculty of Mathematics, Yazd University, Yazd 89195741, Iran \\ ${ }^{2}$ Department of Mathematics, University of Salerno, Via Ponte Don Melillo, 84084 Fisciano, Italy \\ ${ }^{3}$ School of Information Science \& Technology, East China Normal University, Shanghai 200241, China \\ Correspondence should be addressed to M. R. Hooshmandasl; hooshmandasl@yazduni.ac.ir
}

Received 7 August 2013; Accepted 17 August 2013

Academic Editor: Cristian Toma

Copyright (C) 2013 M. H. Heydari et al. This is an open access article distributed under the Creative Commons Attribution License, which permits unrestricted use, distribution, and reproduction in any medium, provided the original work is properly cited.

\begin{abstract}
A new operational matrix of fractional order integration for Legendre wavelets is derived. Block pulse functions and collocation method are employed to derive a general procedure for forming this matrix. Moreover, a computational method based on wavelet expansion together with this operational matrix is proposed to obtain approximate solution of the fractional population growth model of a species within a closed system. The main characteristic of the new approach is to convert the problem under study to a nonlinear algebraic equation.
\end{abstract}

\section{Introduction}

In recent years, fractional calculus and differential equations have found enormous applications in mathematics, physics, chemistry, and engineering because of the fact that a realistic modeling of a physical phenomenon having dependence not only at the time instant but also on the previous time history can be successfully achieved by using fractional calculus. The applications of the fractional calculus have been demonstrated by many authors. For examples, it has been applied to model the nonlinear oscillation of earthquakes, fluid-dynamic traffic, frequency dependent damping behavior of many viscoelastic materials, continuum and statistical mechanics, colored noise, solid mechanics, economics, signal processing, and control theory [1-5]. However, during the last decade fractional calculus has attracted much more attention of physicists and mathematicians. Due to the increasing applications, some schemes have been proposed to solve fractional differential equations. The most frequently used methods are Adomian decomposition method (ADM) [6, 7], homotopy perturbation method [8], homotopy analysis method [9], variational iteration method (VIM) [10], fractional differential transform method (FDTM) [11, 12], fractional difference method (FDM) [13], power series method [14], generalized block pulse operational matrix method [15], and Laplace transform method [16]. Also, recently the Haar wavelets [17], Legendre wavelets [18, 19], and the Chebyshev wavelets of first kind [20-23] and second kind [24] have been developed to solve the fractional differential equations. It is worth noting that wavelets are localized functions, which are the basis for energy-bounded functions and in particular for $L^{2}(R)$, so that localized pulse problems can be easily approached and analyzed [25-28].

Approximation by orthogonal family of basis functions has found wide applications in science and engineering. The most commonly used orthogonal families of functions in recent years are sine-cosine functions, block pulse functions, Legendre, Chebyshev, and Laguerre polynomials and also orthogonal wavelets, for example Haar, Legendre, Chebyshev, and CAS wavelets. The main advantages of using an orthogonal basis is that the problem under consideration reduces to a system of linear or nonlinear algebraic system equations [18]; thus this act not only simplifies the problem enormously but also speeds up the computation work during the implementation. This work can be done by truncating the series expansion in orthogonal basis function for the unknown solution of the problem and using the operational matrices [29]. There are two main approaches for numerical solution of fractional differential equations. 
One approach is based on using the operational matrix of fractional derivative to reduce the problem under consideration into a system of algebraic equations and solving this system to obtain the numerical solution of the problem. Another useful approach is based on converting the underlying fractional differential equations into fractional integral equations, and using the operational matrix of fractional integration, to eliminate the integral operations and reducing the problem into solving a system of algebraic equations. The operational matrix of fractional Riemann-Liouville integration is given by

$$
I^{\alpha} \Psi(x) \simeq P^{\alpha} \Psi(x)
$$

where $\Psi(x)=\left[\psi_{1}(x), \psi_{2}(x), \ldots, \psi_{\widehat{m}}\right]^{T}$, in which $\psi_{i}(x)(i=$ $1,2, \ldots, \widehat{m})$ are orthogonal basis functions which are orthogonal with respect to a specific weight function on a certain interval $[a, b]$ and $P^{\alpha}$ is the operational matrix of fractional integration of $\Psi(x)$. Notice that $P^{\alpha}$ is a constant $\widehat{m} \times \widehat{m}$ matrix and $\alpha$ is an arbitrary positive constant.

In view of successful application of wavelet operational matrices in numerical solution of integral and differential equations, together with the characteristics of wavelet functions, we believe that they can be applicable in solving fractional population growth model. In this paper, the operational matrix of fractional order integrations for Legendre wavelets is derived, and a general procedure based on collocation method and block Pulse functions (BPFs) for forming this matrix is presented. Then, by using this matrix a computational method for solving fractional population growth model in a closed system is proposed. This paper is organized as follows. In Section 2, some necessary definitions of the fractional calculus are reviewed. In Section 3, the Legendre wavelets with some of their properties are presented. In Section 4, the proposed method for solving fractional population growth model in a closed system is described. Finally a conclusion is drawn in Section 5.

\section{Preliminaries}

In this section, we present some notations, definitions, and preliminary facts that will be used further in this paper.

The Riemann-Liouville fractional integral operator $I^{\alpha}$ of order $\alpha \geq 0$ on the usual Lebesgue space $L^{1}[0, b]$ is given by [30]

$$
\left(I^{\alpha} u\right)(x)= \begin{cases}\frac{1}{\Gamma(\alpha)} \int_{0}^{x}(x-s)^{\alpha-1} u(s) d s, & \alpha>0 \\ u(x), & \alpha=0 .\end{cases}
$$

The Riemann-Liouville fractional derivative of order $\alpha>0$ is normally defined as

$$
D^{\alpha} u(x)=\left(\frac{d}{d x}\right)^{m} I^{m-\alpha} u(x), \quad(m-1<\alpha \leq m)
$$

where $m$ is an integer.
The fractional derivative of order $\alpha>0$ in the Caputo sense is given by [30]

$$
\begin{array}{r}
D_{*}^{\alpha} u(x)=\frac{1}{\Gamma(m-\alpha)} \int_{0}^{x}(x-s)^{m-\alpha-1} u^{(m)}(s) d s \\
(m-1<\alpha \leq m),
\end{array}
$$

where $m$ is an integer, $x>0$, and $u^{(m)} \in L^{1}[0, b]$.

The useful relation between the Riemann-Liouville operator and Caputo operator is given by the following expression:

$$
I^{\alpha} D_{*}^{\alpha} u(x)=u(x)-\sum_{k=0}^{m-1} u^{(k)}\left(0^{+}\right) \frac{x^{k}}{k !}, \quad(m-1<\alpha \leq m),
$$

where $m$ is an integer, $x>0$, and $u^{(m)} \in L^{1}[0, b]$.

\section{The Legendre Wavelets}

In this section, we briefly present some properties of Legendre wavelets.

3.1. Constructing the Legendre Wavelets. Here we introduce a process to construct the Legendre wavelets on the unit interval $[0,1]$, using recursive wavelet construction which has been proposed in $[31,32]$ for piecewise polynomials on $[0,1]$. For this purpose, we first introduce some notations. Throughout this work, $\mathbb{N}$ denotes the set of all natural numbers, $\mathbb{N}_{0}=\mathbb{N} \cup\{0\}$ and $\mathbb{Z}_{\mu}=\{0,1, \ldots, \mu-1\}$, for a positive integer $\mu$.

For an integer $\mu>1$, we consider the following contractive mappings on the interval $I=[0,1]$ :

$$
\psi_{\epsilon}(t)=\frac{t+\epsilon}{\mu}, \quad t \in[0,1], \epsilon \in \mathbb{Z}_{\mu} .
$$

It is obvious that the mappings $\left\{\psi_{\epsilon}\right\}$ satisfy the following properties:

$$
\begin{gathered}
\psi_{\epsilon}(I) \subset I, \quad \forall \epsilon \in \mathbb{Z}_{\mu}, \\
\bigcup_{\epsilon \in \mathbb{Z}_{\mu}} \psi_{\epsilon}(I)=I .
\end{gathered}
$$

Now, let $F_{0}$ denote the finite dimensional linear space on $[0,1]$ that is spanned by the Legendre polynomials $P_{0}(2 x-$ $1), P_{1}(2 x-1), \ldots$, and $P_{M-1}(2 x-1)$, where $M \in \mathbb{N}$ and $P_{m}$ are the Legendre polynomials of degree $m$, namely,

$$
F_{0}=\operatorname{span}\left\{P_{m}(2 x-1) \mid x \in[0,1], m \in \mathbb{Z}_{\mu}\right\} .
$$

It is well known that the Legendre polynomials $P_{m}$ are orthogonal with respect to the weight function $w(x)=1$ on the interval $[-1,1]$.

In order to construct an orthonormal basis for $L^{2}[0,1]$, for each $\epsilon \in \mathbb{Z}_{\mu}$ we define an isometry $T_{\epsilon}$ on $L^{2}[0,1]$ as follows:

$$
\left(T_{\epsilon} f\right)(x)= \begin{cases}\sqrt{\mu} f\left(\psi_{\epsilon}^{-1}(x)\right), & x \in \psi_{\epsilon}(I), \\ 0, & x \notin \psi_{\epsilon}(I) .\end{cases}
$$


Starting from the space $F_{0}$, we define a sequence of spaces $\left\{F_{k} \mid k \in \mathbb{N}_{0}\right\}$ using the recurrence formula

$$
F_{k+1}=\bigoplus_{\epsilon \in \mathbb{Z}_{\mu}} T_{\epsilon} F_{k}, \quad k \in \mathbb{N}_{0}
$$

where $\oplus$ denotes the direct sum; that is, if $A$ and $B$ are two subspaces of $L^{2}[0,1]$ with $A \cap B=\{0\}$, then

$$
A \oplus B=\{f+g: f \in A, g \in B\} .
$$

The sequence of spaces $\left\{F_{k} \mid k \in \mathbb{N}_{0}\right\}$ is nested, that is, [32]:

$$
\begin{gathered}
F_{0} \subset F_{1} \subset \cdots \subset F_{k} \subset F_{k+1} \subset \cdots, \\
\operatorname{dim} F_{k}=M \mu^{k}, \quad k \in \mathbb{N}_{0} .
\end{gathered}
$$

Moreover, similar to Theorem 2.4 in [33], it can be proved that

$$
\overline{\bigcup_{k=0}^{\infty} F_{k}}=L^{2}[0,1]
$$

Now, we construct an orthonormal basis for each of the spaces $F_{k}$. We first notice that

$$
G_{0}=\left\{\sqrt{2 m+1} P_{m}(2 x-1) \mid x \in[0,1], m \in \mathbb{Z}_{\mu}\right\}
$$

is an orthonormal basis for $F_{0}$, and moreover for $f(x) \epsilon$ $L^{2}[0,1]$ with compact support and for $\epsilon \neq \epsilon^{\prime}$ we have

$$
\operatorname{supp}\left\{T_{\epsilon} f\right\} \cap \operatorname{supp}\left\{T_{\epsilon^{\prime}} f\right\}=\emptyset, \quad \epsilon \neq \epsilon^{\prime},
$$

where $\operatorname{supp}(f)$ denotes the support of the function $f$. It can be simply seen that [31]

$$
\begin{gathered}
G_{k}=\left\{T_{\epsilon 0} \circ \cdots \circ T_{\epsilon k-1}\left(\sqrt{2 m+1} P_{m}(2 x-1)\right) \mid\right. \\
\left.m \in \mathbb{Z}_{M}, \epsilon_{\ell} \in \mathbb{Z}_{\mu}, \quad \ell \in \mathbb{Z}_{k}\right\}
\end{gathered}
$$

is an orthonormal basis for $F_{k}$, where "o" denotes composition of functions. In other words, if for $n=1,2, \ldots, \mu^{k}, k \in \mathbb{N}$, we set

$$
\begin{aligned}
\psi_{n m}(x) & =\psi(k, m, n, x) \\
& = \begin{cases}\sqrt{2 m+1} \mu^{k / 2} P_{m}\left(2 \mu^{k} x-2 n+1\right), & x \in\left[\frac{n-1}{\mu^{k}}, \frac{n}{\mu^{k}}\right), \\
0, & \text { otherwise, }\end{cases}
\end{aligned}
$$

then $\left\{\psi_{n m}(x) \mid n=1,2, \ldots, \mu^{k}, m \in Z_{M}\right\}$ forms an orthonormal basis for $F_{k}$.

3.2. Function Approximation. A function $f(x)$ defined over $[0,1)$ may be expanded by the Legendre wavelets as

$$
u(x)=\sum_{n=1}^{\infty} \sum_{m=0}^{\infty} c_{n m} \psi_{n m}(x),
$$

where $c_{n m}=\left(u(x), \psi_{n m}(x)\right)$, and $(\cdot, \cdot)$ denotes the inner product. If the infinite series in (18) is truncated, then it can be written as

$$
u(x) \simeq \sum_{n=1}^{\mu^{k}} \sum_{m=0}^{M-1} c_{n m} \psi_{n m}(x)=C^{T} \Psi(x)
$$

where $T$ indicates transposition, $C$ and $\Psi(x)$ are $\widehat{m}=$ $\mu^{k} M$ column vectors which are given by

$$
\begin{gathered}
C=\left[c_{10}, \ldots, c_{1 M-1}\left|c_{20}, \ldots, c_{2 M-1}\right| \cdots \mid c_{\mu^{k} 0}, \ldots, c_{\mu^{k} M-1}\right]^{T}, \\
\Psi(x)=\left[\psi_{10}(x), \ldots, \psi_{1 M-1}(x) \mid \psi_{20}(x), \ldots,\right. \\
\left.\psi_{2 M-1}(x)|\cdots| \psi_{\mu^{k} 0}(x), \ldots, \psi_{\mu^{k} M-1}(x)\right]^{T} .
\end{gathered}
$$

Taking the collocation points

$$
t_{i}=\frac{(2 i-1)}{2 \widehat{m}}, \quad i=1,2, \ldots, \widehat{m},
$$

we define the wavelet matrix $\Phi_{\widehat{m} \times \widehat{m}}$ as

$$
\Phi_{\widehat{m} \times \widehat{m}}=\left[\Psi\left(\frac{1}{2 \widehat{m}}\right), \Psi\left(\frac{3}{2 \widehat{m}}\right), \ldots, \Psi\left(\frac{2 \widehat{m}-1}{2 \widehat{m}}\right)\right] .
$$

Indeed $\Phi_{\widehat{m} \times \widehat{m}}$ has the following form:

$$
\Phi_{\widehat{m} \times \widehat{m}}=\left(\begin{array}{ccccc}
A & 0 & 0 & \ldots & 0 \\
0 & A & 0 & \ldots & 0 \\
0 & 0 & A & \ldots & 0 \\
\vdots & \vdots & \ddots & \ddots & \vdots \\
0 & 0 & \ldots & 0 & A
\end{array}\right),
$$

where $A$ is an $M \times M$ matrix given by 


$$
A=\left(\begin{array}{cccc}
\psi_{10}\left(\frac{1}{2 \widehat{m}}\right) & \psi_{10}\left(\frac{3}{2 \widehat{m}}\right) & \ldots & \psi_{10}\left(\frac{2 \widehat{m}-1}{2 \widehat{m}}\right) \\
\psi_{11}\left(\frac{1}{2 \widehat{m}}\right) & \psi_{11}\left(\frac{3}{2 \widehat{m}}\right) & \ldots & \psi_{11}\left(\frac{2 \widehat{m}-1}{2 \widehat{m}}\right) \\
\vdots & \vdots & \vdots & \vdots \\
\psi_{\mu^{k} M-1}\left(\frac{1}{2 \widehat{m}}\right) & \psi_{\mu^{k} M-1}\left(\frac{3}{2 \widehat{m}}\right) & \ldots & \psi_{\mu^{k} M-1}\left(\frac{2 \widehat{m}-1}{2 \widehat{m}}\right)
\end{array}\right) .
$$

For example, for $\mu=3, k=1, M=2$, the Legendre matrix can be expressed as:

$$
\Phi_{6 \times 6}=\left(\begin{array}{cccccc}
1.7321 & 1.7321 & 0.0 & 0.0 & 0.0 & 0.0 \\
-1.5000 & 1.5000 & 0.0 & 0.0 & 0.0 & 0.0 \\
0.0 & 0.0 & 1.7321 & 1.7321 & 0.0 & 0.0 \\
0.0 & 0.0 & -1.5000 & 1.5000 & 0.0 & 0.0 \\
0.0 & 0.0 & 0.0 & 0.0 & 1.7321 & 1.7321 \\
0.0 & 0.0 & 0.0 & 0.0 & -1.5000 & 1.5000
\end{array}\right)
$$

3.3. Operational Matrix of Fractional Order Integration. The fractional integration of order $\alpha$ of the vector function $\Psi(x)$ can be expressed as

$$
\left(I^{\alpha} \Psi\right)(x) \simeq P^{\alpha} \Psi(x)
$$

where $P^{\alpha}$ is the $\widehat{m} \times \widehat{m}$ operational matrix of fractional integration of order $\alpha$. In the following we obtain an explicit form of the matrix $P$. For this purpose, we need to introduce a new family of basis functions, namely, block pulse functions (BPFs).

We define a $\widehat{m}$-set of BPFs as $[34,35]$

$$
b_{i}(x)= \begin{cases}1, & \frac{i}{\widehat{m}} \leq x<\frac{(i+1)}{\widehat{m}} \\ 0, & \text { otherwise }\end{cases}
$$

where $i=0,1,2, \ldots,(\widehat{m}-1)$.

The functions $b_{i}(x)$ are disjoint and orthogonal.

The Legendre wavelets may be expanded into a $\widehat{m}$-set of BPFs as

$$
\Psi(x) \simeq \Phi_{\widehat{m} \times \widehat{m}} B_{\widehat{m}}(x),
$$

where $B_{\widehat{m}}(x)=\left[b_{0}(x), b_{1}(x), \ldots, b_{i}(x), \ldots, b_{\widehat{m}-1}(x)\right]^{T}$.
In [34], Kilicman et al. have given the block pulse operational matrix of fractional integration $P_{B}^{\alpha}$ as

$$
\left(I^{\alpha} B_{\widehat{m}}\right)(x) \simeq P_{B}^{\alpha} B_{\widehat{m}}(x),
$$

where

$$
P_{B}^{\alpha}=\frac{1}{\widehat{m}^{\alpha}} \frac{1}{\Gamma(\alpha+2)}\left(\begin{array}{ccccc}
1 & \xi_{1} & \xi_{2} & \ldots & \xi_{\widehat{m}-1} \\
0 & 1 & \xi_{1} & \ldots & \xi_{\widehat{m}-2} \\
0 & 0 & 1 & \ldots & \xi_{\widehat{m}-3} \\
0 & 0 & 0 & \ddots & \vdots \\
0 & 0 & 0 & 0 & 1
\end{array}\right)
$$

and $\xi_{i}=(i+1)^{\alpha+1}-2 i^{\alpha+1}+(i-1)^{\alpha+1}$.

Next, we derive the Legendre wavelets operational matrix of fractional integration. By considering (26) and using (28), and (29) we have

$$
\begin{aligned}
\left(I^{\alpha} \Psi\right)(x) & \simeq\left(I^{\alpha} \Phi_{\widehat{m} \times \widehat{m}} B_{\widehat{m}}\right)(x)=\Phi_{\widehat{m} \times \widehat{m}}\left(I^{\alpha} B_{\widehat{m}}\right)(t) \\
& \simeq \Phi_{\widehat{m} \times \widehat{m}} P_{B}^{\alpha} B_{\widehat{m}}(x) .
\end{aligned}
$$

Thus, by considering (28) and (31), we obtain the Legendre wavelets operational matrix of fractional integration as

$$
\left(I^{\alpha} \Psi\right)(x) \simeq \Phi_{\widehat{m} \times \widehat{m}} P_{B}^{\alpha} \Phi_{\widehat{m} \times \widehat{m}}^{-1} .
$$

To illustrate the calculation procedure we choose $\mu=3, k=$ $1, M=2$, and $\alpha=1 / 2$; thus we have: 


$$
P^{(1 / 2)}=\left(\begin{array}{cccccc}
0.43433 & 0.14689 & 0.35988 & -0.069510 & 0.23430 & -0.017626 \\
-0.11016 & 0.17991 & 0.052129 & -0.028562 & 0.013219 & -0.0032248 \\
0.0 & 0.0 & 0.43433 & 0.14689 & 0.35988 & -0.069510 \\
0.0 & 0.0 & -0.11016 & 0.17991 & 0.052129 & -0.028562 \\
0.0 & 0.0 & 0.0 & 0.0 & 0.43433 & 0.14689 \\
0.0 & 0.0 & 0.0 & 0.0 & -0.11016 & 0.17991
\end{array}\right) .
$$

\section{Application for Fractional Population Growth Model}

As we have already mentioned, the fractional order models are more accurate than integer order models; that is, there are more degrees of freedom in the fractional order models. In this section, we will apply Legendre wavelets for solving a fractional population growth model. The model is characterized by the nonlinear fractional Volterra integrodifferential equation [36] as follows:

$$
\begin{array}{r}
D_{*}^{\alpha} p(\bar{t})-\bar{a} p(\bar{t})+\bar{b}[p(\bar{t})]^{2}+\bar{c} p(\bar{t}) \int_{0}^{\bar{t}} p(\tau) d \tau=0, \\
p(0)=p_{0}, \quad 0<\alpha \leq 1,
\end{array}
$$

where $\alpha$ is a constant parameter describing the order of the time fractional derivative, $\bar{a}>0$ is the birth rate coefficient, $\bar{b}>0$ is the crowding coefficient, $\bar{c}>0$ is the toxicity coefficient, $p_{0}$ is the initial population, and $p(\bar{t})$ is the population of identical individuals at time $\bar{t}$ which exhibits crowding and sensitivity to the amount of toxins produced [37]. The coefficient $\bar{c}$ indicates the essential behavior of the population evolution before its level falls to zero in the long run. It is worth mentioning that when the toxicity coefficient is zero, (34) reduces to the well-known logistic equation $[37,38]$. The last term contains the integral which indicates the total metabolism or total amount of toxins produced since time zero. The individual death rate is proportional to this integral, and also the population death rate due to toxicity must include a factor $p$. Due to the fact that the system is closed, the presence of the toxic term always causes the population level falling to zero in the long run, as it will be seen later. The relative size of the sensitivity to toxins, $\bar{c}$, determines the manner in which the population evolves before its extinction. It is worth noting that in case $\alpha=1$, the fractional equation reduces to a classical logistic growth model, so the proposed method can be also applied in this situation. Here we apply the scale time and population by introducing the non-dimensional variables $t=\bar{c} \bar{t} / \bar{b}$ and $u=$ $\bar{b} p / \bar{a}$, to obtain the following non-dimensional problem:

$$
\begin{array}{r}
\kappa D_{*}^{\alpha} u(t)-u(t)+[u(t)]^{2}+u(t) \int_{0}^{t} u(\tau) d \tau=0, \\
u(0)=u_{0}, \quad 0<\alpha \leq 1,
\end{array}
$$

where $u(t)$ is the scaled population of identical individuals at time $t$ and $\kappa=\bar{c} / \bar{a} \bar{b}$ is a prescribed non-dimensional parameter. The only equilibrium solution of (35) is the trivial solution $u(t)=0$, and the analytical solution for $\alpha=1$ is [39]

$$
u(t)=u_{0} \exp \left(\frac{1}{\kappa} \int_{0}^{t}\left(1-u(\tau)-\int_{0}^{\tau} u(s) d s\right) d \tau\right) .
$$

In recent years, several numerical methods have been proposed to solve the classical and fractional population growth model, for instance, the reader is advised to see [36-43] and references therein. Here we use the operational matrix of fractional integration for solving nonlinear fractional integrodifferential population model (35). For this purpose, we first approximate $D_{*}^{\alpha} u(t)$ as

$$
D_{*}^{\alpha} u(t) \simeq U^{T} \Psi(t),
$$

where $U$ is an unknown vector which should be found and $\Psi(t)$ is the vector which is defined in (20).

By using initial condition and (5), we have

$$
u(t) \simeq U^{T} P^{\alpha} \Psi(t)+u_{0} .
$$

Since $\Psi(t) \simeq \Phi_{\widehat{m} \times \widehat{m}} B_{\widehat{m}}(t)$, from (38), we have:

$$
u(t) \simeq U^{T} P^{\alpha} \Phi_{\widehat{m} \times \widehat{m}} B_{\widehat{m}}(t)+u_{0}[1,1, \ldots, 1] B_{\widehat{m}}(t) .
$$

Define

$$
A^{T}=\left[a_{1}, a_{2}, \ldots, a_{\widehat{m}}\right]=U^{T} P^{\alpha} \Phi_{\widehat{m} \times \widehat{m}}+u_{0}[1,1, \ldots, 1] .
$$

By using (38) and (39), we have $u(t) \simeq A^{T} B_{\widehat{m}}(t)$. From (27), we have

$$
[u(t)]^{2} \simeq\left[a_{1}^{2}, a_{2}^{2}, \ldots, a_{\widehat{m}}^{2}\right] B_{\widehat{m}}(t)=\widehat{A}^{T} B_{\widehat{m}}(t) .
$$

Also, we have

$$
\int_{0}^{t} u(\tau) d \tau \simeq A^{T} P_{B} B_{\widehat{m}}(t)=C^{T} B_{\widehat{m}}(t),
$$

where $C^{T}=A^{T} P_{B}$. Now using (27), (39), and (42), we have

$$
u(t) \int_{0}^{t} u(\tau) d \tau \simeq \widetilde{U}^{T} B_{\widehat{m}}(t)
$$

where

$$
\widetilde{U}^{T}=\left[a_{1} c_{1}, a_{2} c_{2}, \ldots, a_{\widehat{m}} c_{\widehat{m}}\right] .
$$

Now by substituting (37), (39), (41) and (43), into (35), we obtain

$$
\left(\bar{k} U^{T} \Phi_{\widehat{m} \times \widehat{m}}-A^{T}+\widetilde{A}^{T}+\widehat{U}^{T}\right) B_{\widehat{m}}(t) \simeq 0
$$




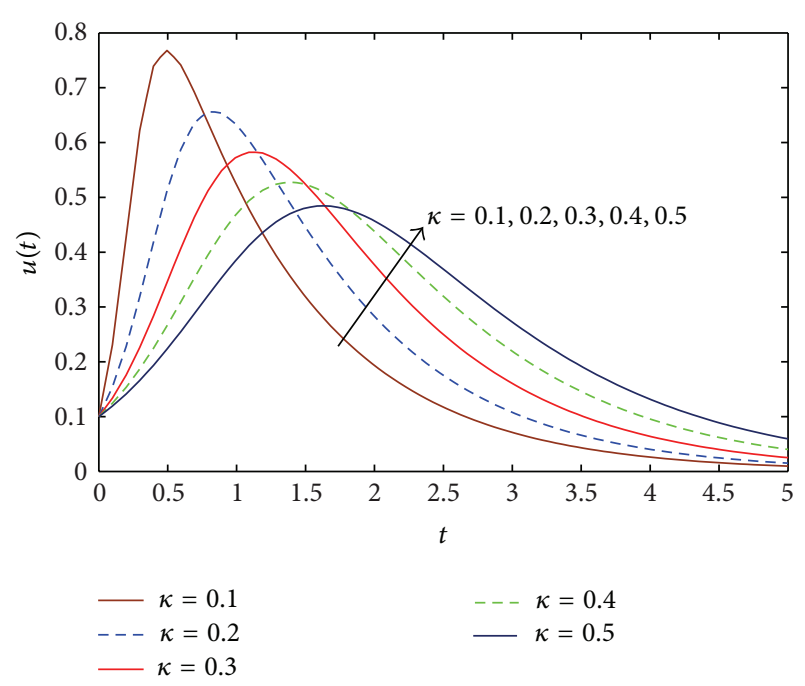

Figure 1: Numerical solutions of the classical population growth model for different values of $\kappa$.

and by replacing $\simeq$ by $=$, we obtain the following system of nonlinear algebraic equations:

$$
\kappa U^{T} \Phi_{\widehat{m} \times \widehat{m}}-A^{T}+\widetilde{A}^{T}+\widehat{U}^{T}=0
$$

Finally by solving this system and determining $A$, we obtain the approximate solution of the problem as $u(t)=A^{T} \Psi(t)$.

As a numerical example, we consider the nonlinear fractional integrodifferential equation (35) with the initial condition $u(0)=0.1$, which is investigated in several papers, for instance see [36-43]. Here our purpose is to study the mathematical behavior of the solution of this fractional population growth model as the order of the fractional derivative changes. In particular, we seek to study the rapid growth along the logistic curve that will reach a peak then slow exponential decayed for different values of $\alpha$. To see the behavior solution of this problem for different values of $\alpha$, we will take advantage of the proposed method and consider the following two special cases.

Case 1. We investigate the classical population growth model $(\alpha=1)$ for some different small values $\kappa$. The behavior of the numerical solutions for $\widehat{m}=162(\mu=3, k=3$, and $M=$ 6 ) is shown in Figure 1. From Figure 1 it can be seen that as $\kappa$ increases, the amplitude of $u(t)$ decreases, whereas the exponential decay increases.

Case 2. In this case we investigate the fractional population growth model (35) for different values of $\alpha$ and $\kappa$.

From Figures 2, 3, and 4 it can be simply seen that as the order of the fractional derivative decreases, the amplitude of $u(t)$ decreases, whereas the exponential decay increases and also it can be concluded that as $\kappa$ increases, the maximum of $u\left(t^{*}\right)$ of $u(t)$ decreases. This tendency is similar to the case $\alpha=1$, which we have already mentioned.

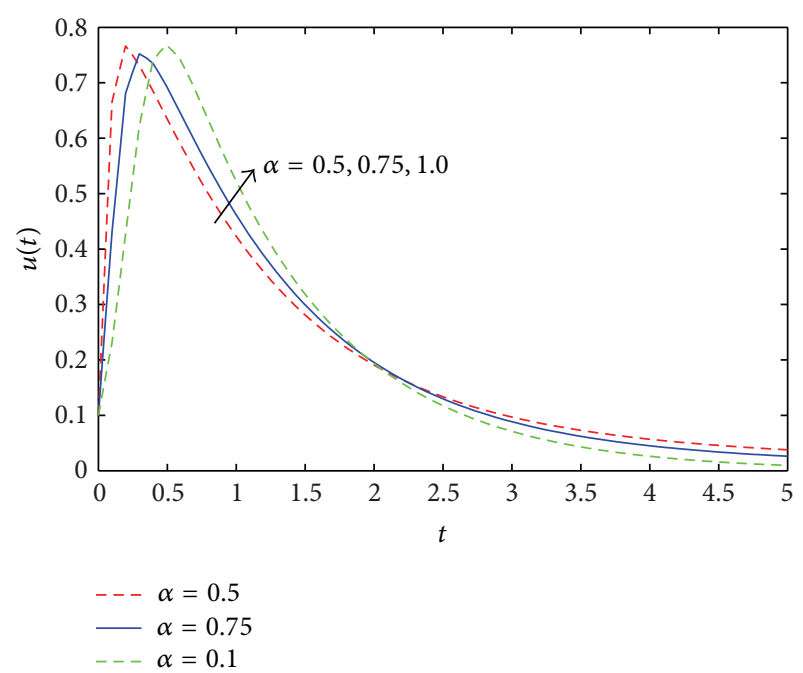

FIGURE 2: Numerical solutions of the fractional population growth model for $\kappa=0.1$.

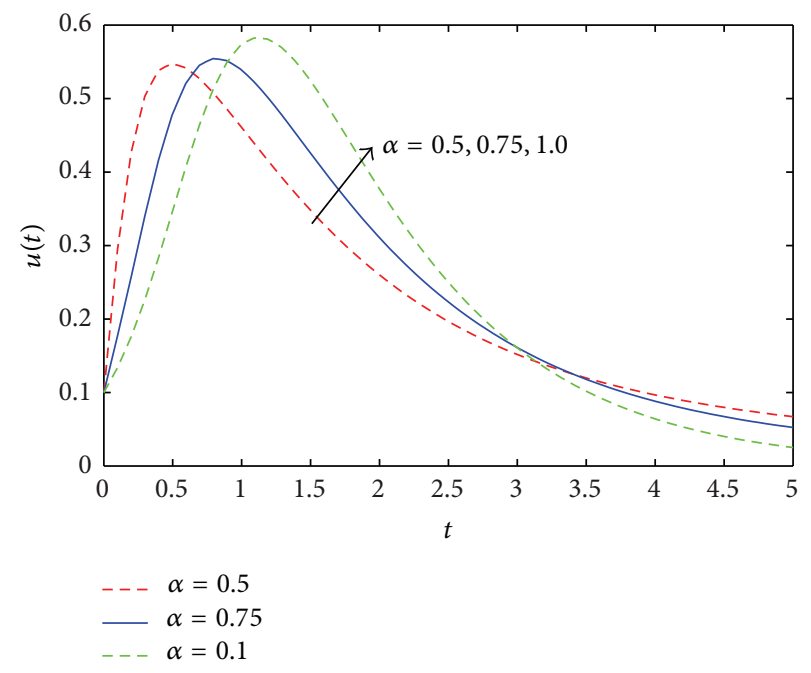

FIGURE 3: Numerical solutions of the fractional population growth model for $\kappa=0.3$.

\section{Conclusion}

In this paper, the operational matrix of fractional order integration for Legendre wavelets was derived. Block pulse functions and collocation method were employed to derive a general procedure for forming this matrix. Moreover, a wavelet expansion together with this operational matrix was used to obtain approximate solution of the fractional population growth model of a species within a closed system. The main characteristic of the new approach is to convert the problem under study to a system of nonlinear algebraic equations by introducing the operational matrix of fractional integration for these basis functions. Analysis of the behavior of the model showed that it increases rapidly along the logistic curve followed by a slow exponential decay after reaching a maximum point, and also when the order of 


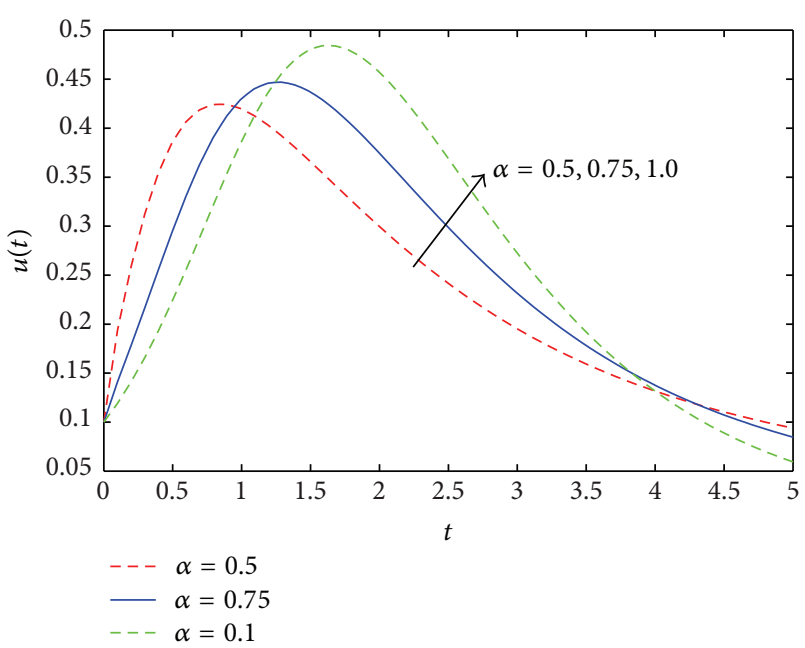

FIGURE 4: Numerical solutions of the fractional population growth model for $\kappa=0.5$.

the fractional derivative $\alpha$ decreases, the amplitude of the solution decreases, whereas the exponential decay increases.

\section{Acknowledgment}

This work was supported in part by the National Natural Science Foundation of China under the project Grant nos. 61272402, 61070214, and 60873264 .

\section{References}

[1] J. H. He, "Nonlinear oscillation with fractional derivative and its applications," in Proceedings of the International Conference on Vibrating Engineering, vol. 98, pp. 288-291, Dalian, China, 1998.

[2] R. L. Bagley and P. J. Torvik, "A theoretical basis for the application of fractional calculus to viscoelasticity," Journal of Rheology, vol. 27, no. 3, pp. 201-210, 1983.

[3] F. Mainardi, "Fractional calculus: some basic problems in continuum and statistical mechanics," in Fractals and Fractional Calculus in Continuum Mechanics, A. Carpinteri and F. Mainardi, Eds., vol. 378, pp. 291-348, Springer, New York, NY, USA, 1997.

[4] Y. A. Rossikhin and M. V. Shitikova, "Applications of fractional calculus to dynamic problems of linear and nonlinear hereditary mechanics of solids," Applied Mechanics Reviews, vol. 50, pp. 15-67, 1997.

[5] R. T. Baillie, "Long memory processes and fractional integration in econometrics," Journal of Econometrics, vol. 73, no. 1, pp. 559, 1996.

[6] S. Momani and Z. Odibat, "Numerical approach to differential equations of fractional order," Journal of Computational and Applied Mathematics, vol. 207, no. 1, pp. 96-110, 2007.

[7] S. A. El-Wakil, A. Elhanbaly, and M. A. Abdou, "Adomian decomposition method for solving fractional nonlinear differential equations," Applied Mathematics and Computation, vol. 182, no. 1, pp. 313-324, 2006.

[8] N. H. Sweilam, M. M. Khader, and R. F. Al-Bar, "Numerical studies for a multi-order fractional differential equation," Physics Letters A, vol. 371, no. 1-2, pp. 26-33, 2007.
[9] I. Hashim, O. Abdulaziz, and S. Momani, "Homotopy analysis method for fractional IVPs," Communications in Nonlinear Science and Numerical Simulation, vol. 14, no. 3, pp. 674-684, 2009.

[10] S. Das, "Analytical solution of a fractional diffusion equation by variational iteration method," Computers \& Mathematics with Applications, vol. 57, no. 3, pp. 483-487, 2009.

[11] A. Arikoglu and I. Ozkol, "Solution of fractional integrodifferential equations by using fractional differential transform method," Chaos, Solitons and Fractals, vol. 40, no. 2, pp. 521-529, 2009.

[12] V. S. Ertürk and S. Momani, "Solving systems of fractional differential equations using differential transform method," Journal of Computational and Applied Mathematics, vol. 215, no. 1, pp. 142-151, 2008.

[13] M. M. Meerschaert and C. Tadjeran, "Finite difference approximations for two-sided space-fractional partial differential equations," Applied Numerical Mathematics, vol. 56, no. 1, pp. 80-90, 2006.

[14] Z. M. Odibat and N. T. Shawagfeh, "Generalized Taylor's formula," Applied Mathematics and Computation, vol. 186, no. 1, pp. 286-293, 2007.

[15] Y. Li and N. Sun, "Numerical solution of fractional differential equations using the generalized block pulse operational matrix," Computers \& Mathematics with Applications, vol. 62, no. 3, pp. 1046-1054, 2011.

[16] I. Podlubny, "The Laplace transform method for linear differential equations of the fractional order," UEF-02-94, Institute of Experimental Physics, Slovak Academy of Sciences, Kosice, Slovakia, 1994.

[17] Y. Li and W. Zhao, "Haar wavelet operational matrix of fractional order integration and its applications in solving the fractional order differential equations," Applied Mathematics and Computation, vol. 216, no. 8, pp. 2276-2285, 2010.

[18] M. Rehman and R. Ali Khan, "The Legendre wavelet method for solving fractional differential equations," Communications in Nonlinear Science and Numerical Simulation, vol. 16, no. 11, pp. 4163-4173, 2011.

[19] M. H. Heydari, M. R. Hooshmandasl, F. M. M. Ghaini, and F. Fereidouni, "Two-dimensional legendre wavelets forsolving fractional poisson equation with dirichlet boundary conditions," Engineering Analysis With Boundary Elements, vol. 37, pp. 1331-1338, 2013.

[20] Y. Li, "Solving a nonlinear fractional differential equation using Chebyshev wavelets," Communications in Nonlinear Science and Numerical Simulation, vol. 15, no. 9, pp. 2284-2292, 2010.

[21] M. H. Heydari, M. R. Hooshmandasl, F. M. M. Ghaini, and F. Mohammadi, "Wavelet collocation method for solving multi order fractional differential equations," Journal of Applied Mathematics, vol. 2012, Article ID 542401, 19 pages, 2012.

[22] M. H. Heydari, M. R. Hooshmandasl, F. Mohammadi, and C. Cattani, "Wavelets method for solving systems of nonlinear singular fractional volterra integro-differential equations," Communications in Nonlinear Science and Numerical Simulation, vol. 19, no. 1, pp. 37-48, 2014

[23] M. R. Hooshmandasl, M. H. Heydari, and F. M. M. Ghaini, "Numerical solution of the one-dimensional heat equation by using chebyshev wavelets method," Applied and Computational Mathematics, vol. 1, no. 6, Article ID 42401, 19 pages, 2012.

[24] Y. Wang and Q. Fan, "The second kind Chebyshev wavelet method for solving fractional differential equations," Applied 
Mathematics and Computation, vol. 218, no. 17, pp. 8592-8601, 2012.

[25] C. Cattani, "Fractional calculus and Shannon wavelet," Mathematical Problems in Engineering, vol. 2012, Article ID 502812, 26 pages, 2012.

[26] C. Cattani, "Shannon wavelets for the solution of integrodifferential equations," Mathematical Problems in Engineering, vol. 2010, Article ID 408418, 22 pages, 2010.

[27] C. Cattani and A. Kudreyko, "Harmonic wavelet method towards solution of the Fredholm type integral equations of the second kind," Applied Mathematics and Computation, vol. 215, no. 12, pp. 4164-4171, 2010.

[28] C. Cattani, "Shannon wavelets theory," Mathematical Problems in Engineering, vol. 2008, Article ID 164808, 24 pages, 2008.

[29] J. Biazar and H. Ebrahimi, "Chebyshev wavelets approach for nonlinear systems of Volterra integral equations," Computers \& Mathematics with Applications, vol. 63, no. 3, pp. 608-616, 2012.

[30] I. Podlubny, Fractional Differential Equations, vol. 198, Academic Press, San Diego, Calif, USA, 1999.

[31] Y. Shen and W. Lin, "Collocation method for the natural boundary integral equation," Applied Mathematics Letters, vol. 19, no. 11, pp. 1278-1285, 2006.

[32] C. A. Micchelli and Y. Xu, "Reconstruction and decomposition algorithms for biorthogonal multiwavelets," Multidimensional Systems and Signal Processing, vol. 8, no. 1-2, pp. 31-69, 1997.

[33] C. A. Micchelli and Y. Xu, "Using the matrix refinement equation for the construction of wavelets on invariant sets," Applied and Computational Harmonic Analysis, vol. 1, no. 4, pp. 391-401, 1994.

[34] A. Kilicman, A. Zhour, and Z. A. Aziz, "Kronecker operational matrices for fractional calculus and some applications," Applied Mathematics and Computation, vol. 187, no. 1, pp. 250-265, 2007.

[35] M. H. Heydari, M. R. Hooshmandasl, and F. M. M. Ghaini, "A good approximate solution for lienard equation in a large interval using block pulse functions," Journal of Mathematical Extension, vol. 7, no. 1, pp. 17-32, 2013.

[36] H. Xu, "Analytical approximations for a population growth model with fractional order," Communications in Nonlinear Science and Numerical Simulation, vol. 14, no. 5, pp. 1978-1983, 2009.

[37] K. G. TeBeest, "Numerical and analytical solutions of Volterra's population model," SIAM Review, vol. 39, no. 3, pp. 484-493, 1997.

[38] F. M. Scudo, "Vito Volterra and theoretical ecology," Theoretical Population Biology, vol. 2, pp. 1-23, 1971.

[39] K. Parand, A. R. Rezaei, and A. Taghavi, "Numerical approximations for population growth model by rational chebyshev and hermite functions collocation approach: a comparison," Mathematical Methods in the Applied Sciences, vol. 33, no. 17, pp. 2076-2086, 2010.

[40] A.-M. Wazwaz, "Analytical approximations and Padé approximants for Volterra's population model," Applied Mathematics and Computation, vol. 100, no. 1, pp. 13-25, 1999.

[41] K. Al-Khaled, "Numerical approximations for population growth models," Applied Mathematics and Computation, vol. 160, no. 3, pp. 865-873, 2005.

[42] K. Al-Khaled, "Analytical approximations for a population growth model with fractional order," Communications in Nonlinear Science and Numerical Simulation, vol. 14, pp. 1978-1983.
[43] K. Krishnaveni and S. B. K. Kannan, "Approximate analytical solution for fractional population growth model," International Journal of Engineering and Technology, vol. 5, no. 3, pp. 28322836, 2013. 


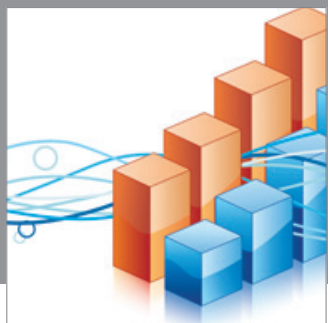

Advances in

Operations Research

mansans

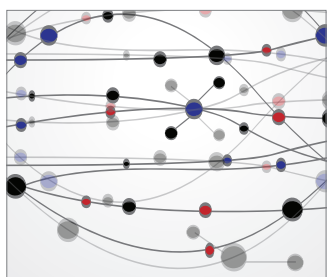

The Scientific World Journal
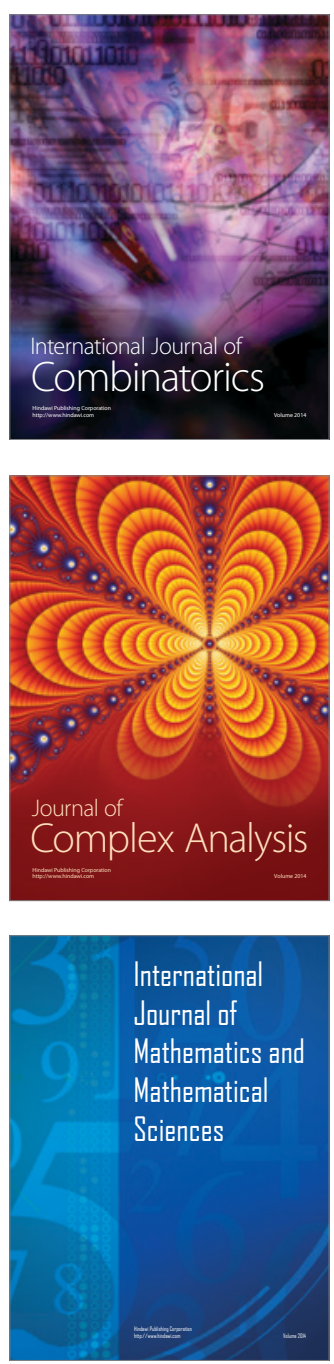
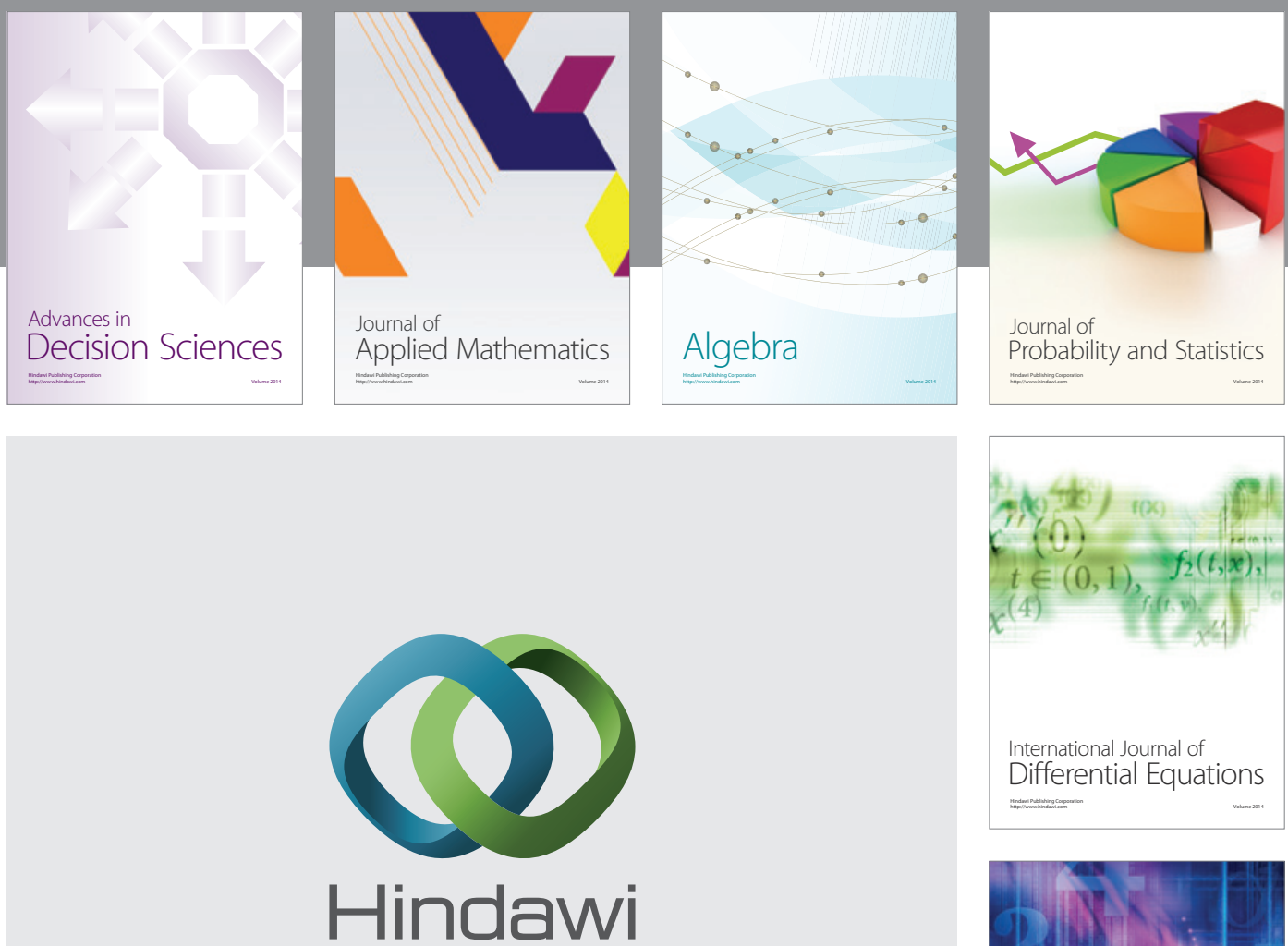

Submit your manuscripts at http://www.hindawi.com
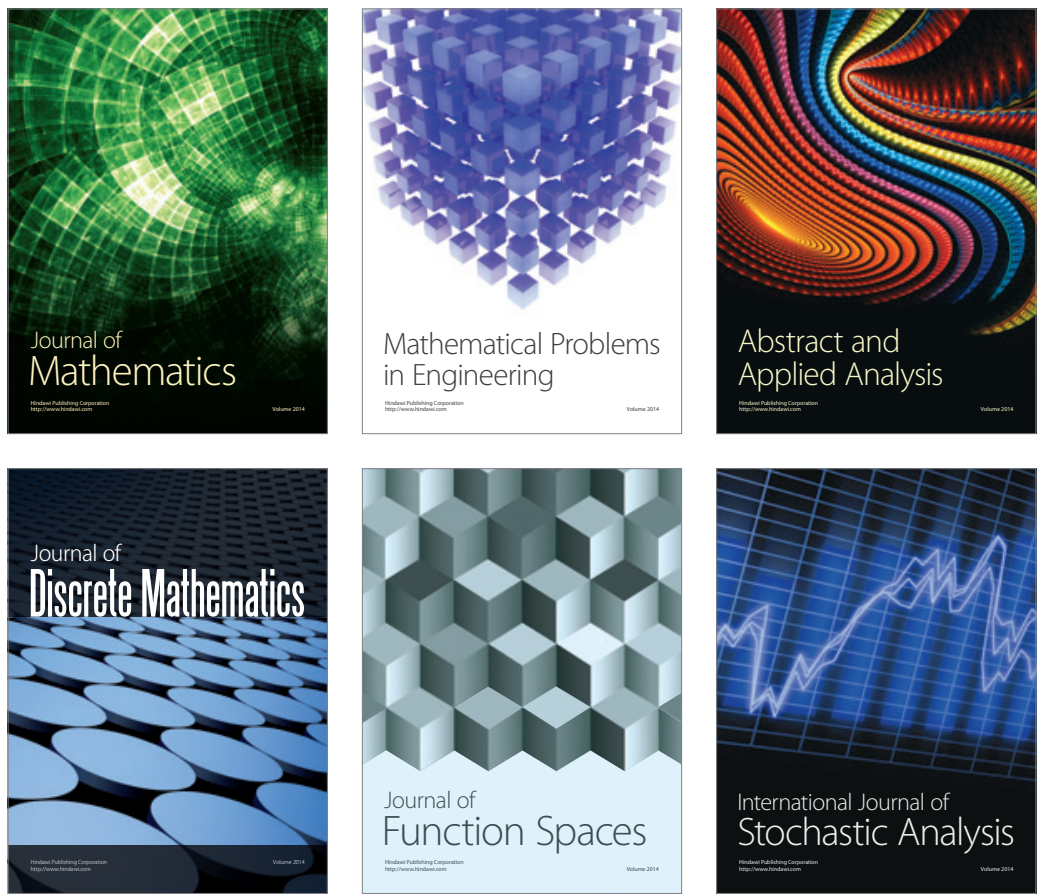

Journal of

Function Spaces

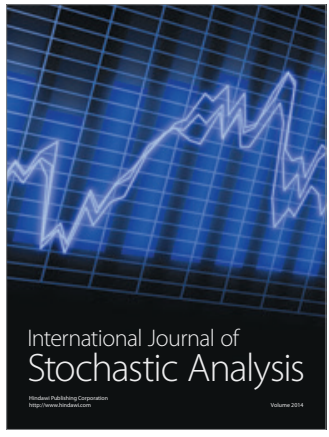

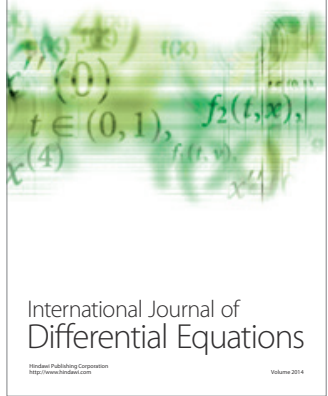
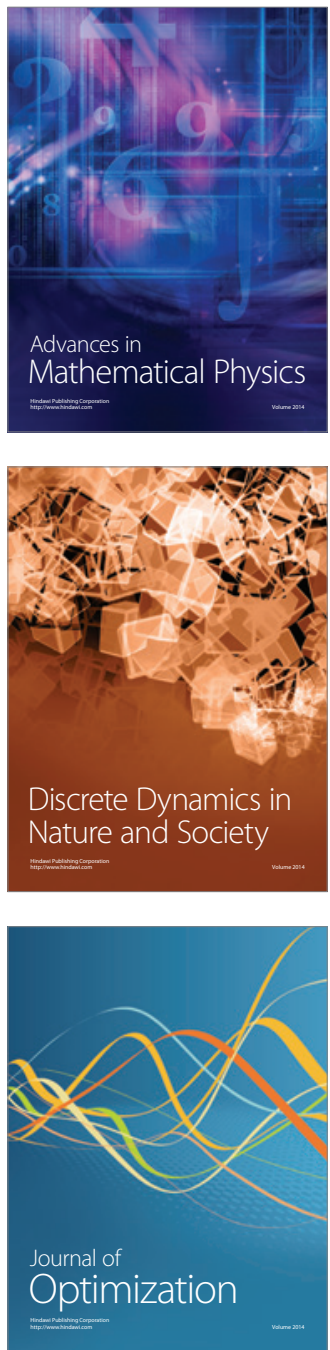